\title{
Plasma microRNA-320, microRNA-let-7e and microRNA-21 as novel potential biomarkers for the detection of retinoblastoma
}

\author{
SHAN-SHAN LIU ${ }^{1,2}$, YU-SHI WANG ${ }^{3,4}$, YAN-FENG SUN $^{2}$, LI-XIA MIAO ${ }^{2}$, \\ JUN WANG ${ }^{2}$, YAN-SHAN LI ${ }^{2}$, HONG-YAN LIU ${ }^{2}$ and QIU-LING LIU ${ }^{2}$ \\ ${ }^{1}$ Graduate Division, Xinxiang Medical University, Xinxiang, Henan 453003; ${ }^{2}$ Department of Pediatrics, \\ General Hospital of Chinese People's Armed Police Forces, Beijing 100039; \\ ${ }^{3}$ Beijing Institute of Radiation Medicine, Beijing 100850; ${ }^{4}$ College of Life Sciences, \\ Jilin University, Changchun, Jilin 130012, P.R. China
}

Received January 03, 2014; Accepted February 21, 2014

DOI: $10.3892 /$ br.2014.246

\begin{abstract}
Retinoblastoma (RB) is a childhood malignancy caused by inactivation of the $R B$ gene, with neuron-specific enolase (NSE) levels considered as its diagnostic marker. MicroRNAs (miRNAs) have been proven to play a significant role in multiple physiological and pathological processes and several miRNAs were identified as tumor biomarkers in recent studies. In the present study, 65 plasma samples were collected from RB patients and 65 samples from healthy individuals to serve as controls. The miRNA levels were measured via quantitative reverse transcription-polymerase chain reaction and their association with RB was assessed by statistical data analysis and receiver operating characteristic curves. Plasma miRNA (miR)-320, miR-let-7e and miR-21 levels were downregulated in the patient samples, the areas under the curves (AUCs) were 0.548-0.660, whereas the AUCs of combined classifiers were $\geq 0.990$. The plasma miRNA levels, particularly of miR-320, were found to be of value in RB diagnosis and may be considered as novel diagnostic biomarkers.
\end{abstract}

\section{Introduction}

Retinoblastoma ( $\mathrm{RB}$ ) is the most common childhood malignancy, with a relative incidence of $1 / 15,000-20,000$ live births annually. The inactivation of the $R B$ gene is considered as the initiating event in this disease (1). Delayed diagnosis and treatment contribute to the exacerbation and migration of RB (2). Thus, a timely and accurate diagnosis is required for earlier treatment, which may increase the cure and survival rates.

Correspondence to: Professor Qiu-Ling Liu, Department of Pediatrics, General Hospital of Chinese People's Armed Police Forces, 69 Yonding Road, Haidian, Beijing 100039, P.R. China E-mail:wj670@vip.sina.com

Key words: retinoblastoma, microRNA, plasma, biomarker
Imaging techniques are widely used for the diagnosis of $\mathrm{RB}$ and images of the tumor tissue may confirm the diagnosis of RB via ophthalmoscopy, ultrasonography, computed tomography and magnetic resonance imaging (2). As a diagnostic marker for small-cell lung cancer and neuroblastoma, neuron-specific enolase (NSE) was found to be significantly elevated in the serum of RB patients and is considered to be a clinical diagnostic indicator (3-6).

MicroRNAs (miRNAs) are a class of mature non-coding single-strand RNAs with a length of 22 nucleotides, which play significant roles in multiple physiological and pathological processes, particularly in tumor development and exacerbation $(7,8)$. An increasing number of studies demonstrate that miRNA expression profiles may be specific to certain types of cancer and tumor-derived miRNAs may be stably detected in the plasma or serum. These findings highlight the potential of circulating miRNAs as biomarkers for the diagnosis of cancer (9-12).

miRNA (miR)-let-7e, a member of the let-7 family, was found to be highly associated with the development and progression of RB. A low level of miR-let-7e contributes to the overexpression of the high-mobility group A1 (HMG A1) and high-mobility group A2 (HMG A2) proteins in RB cells, which are considered as promoters of RB (13). The downregulation of the tumor suppressor miR-let-7e was identified as a biomarker in lung and gastric cancers, uterine leiomyoma and pituitary adenomas (14-17). miR-21 was the first miRNA identified as a diagnostic biomarker, due to its elevated levels in diffuse large B-cell lymphoma (18). miR-320 was suggested to act as a tumor suppressor by inhibiting $\beta$-catenin expression via binding to the 3 '-untranslated region of $\beta$-catenin mRNA in prostate cancer (19). However, it was previously demonstrated that the levels of miR-320 and miR-let-7e were significantly higher in RB compared to those in the normal human retina, according to the results of a miRNA microarray assay (20). Taking into consideration the results of that microarray assay and the fact that miR-21 has been investigated in several types of cancer as a biomarker (21-24), we hypothesized that miR-let-7e, miR-21 and miR-320 may serve as non-invasive circulating biomarkers for the diagnosis of RB. The expression of these 
Table I. Primers used for qRT-PCR.

\begin{tabular}{ll}
\hline Primers & \multicolumn{1}{c}{ Sequences (5'-3') } \\
\hline RT & GCGAGCACAGAATTAATACGACTC \\
ACTATAGG(T)18VN \\
F6
\end{tabular}

qRT-PCR, reverse transcription-quantitative polymerase chain reaction; miR, microRNA.

3 plasma miRNAs and the serum NSE levels were measured in $\mathrm{RB}$ patients and control subjects matched to the patients by age and gender.

\section{Materials and methods}

Patients and samples. Blood samples were collected from consenting individuals according to protocols approved by the Institutional Review Board of the General Hospital of the Chinese People's Armed Police Forces (Beijing, China). Between March, 2012 and June, 2013, a total of 65 patients with RB who had not received any prior treatment and 65 healthy age- and gender-matched controls were enrolled in this study. All the samples were collected once informed consent was obtained from the patients or the legal guardian.

Sample processing and total RNA extraction. Cell-free plasma was isolated via a two-step protocol $(2,500 \mathrm{rpm}$ at room temperature for $10 \mathrm{~min}$ and $14,000 \mathrm{x} \mathrm{g}$ at $4^{\circ} \mathrm{C}$ for $10 \mathrm{~min}$ ) within $2 \mathrm{~h}$ after collection to prevent the contamination of cellular nucleic acids. The resulting plasma was transferred to new tubes and stored at $-80^{\circ} \mathrm{C}$. Total RNA was extracted from $300 \mu \mathrm{l}$ plasma with the mirVana ${ }^{\mathrm{TM}}$ PARIS $^{\mathrm{TM}}$ kit (Ambion, Inc., Foster City, CA, USA) according to the manufacturer's instructions and eluted with $50 \mu$ l elution solution pre-heated at $95^{\circ} \mathrm{C}$. The RNA quality and concentration was assessed with a K5500 spectrophotometer (Beijing Kaiao Technology Development Co., Ltd., Beijing, China). The concentration of the RNA extracted from plasma was 3.9-18.3 ng/ $\mu \mathrm{l}$.

Quantitative reverse transcription-polymerase chain reaction ( $q R T-P C R$ ). Total RNA was polyadenylated by poly(A) polymerase (New England BioLabs, Inc., Ipswich, MA, USA) and reverse-transcribed to cDNA with the Promega reverse transcription kit (Promega, Madison, WI, USA) according to the manufacturer's instructions. The reaction mixture for reverse transcription contained $8 \mu \mathrm{l}$ RNA extract, $2 \mu \mathrm{l}$ reverse transcription primer $(1 \mu \mathrm{g} / \mu \mathrm{l}), 8 \mu \mathrm{l}$ Improm-II ${ }^{\mathrm{TM}} 5 \mathrm{X}$ reaction buffer, $4.8 \mu \mathrm{l} \mathrm{MgCl}_{2}$ (25 mmol/l), $2 \mu \mathrm{l}$ dNTPs (10 and
Table II. Clinical characteristics of the retinoblastoma patients and healthy control subjects.

\begin{tabular}{|c|c|c|c|}
\hline Characteristics & $\begin{array}{l}\text { Cases } \\
(n=65)\end{array}$ & $\begin{array}{l}\text { Controls } \\
(\mathrm{n}=65)\end{array}$ & P-value \\
\hline $\begin{array}{l}\text { Average age, months } \\
(\text { mean } \pm \text { SD) }\end{array}$ & $24.6 \pm 16.5$ & $27.92 \pm 12.03$ & $0.196^{\mathrm{a}}$ \\
\hline Gender, n (\%) & & & $1.000^{\mathrm{b}}$ \\
\hline Male & $39(60.0)$ & $31(47.7)$ & \\
\hline Female & $26(40.0)$ & $34(52.3)$ & \\
\hline \multicolumn{4}{|l|}{ Laterality, n (\%) } \\
\hline Unilateral & $45(69.2)$ & $\mathrm{N} / \mathrm{A}$ & \\
\hline Bilateral & $20(30.8)$ & N/A & \\
\hline \multicolumn{4}{|l|}{ IIRC clinical stage, $n$} \\
\hline Group A-C & 12 & $\mathrm{~N} / \mathrm{A}$ & \\
\hline Group D-E & 53 & N/A & \\
\hline $\begin{array}{l}\text { NSE level, ng/mL } \\
(\text { mean } \pm \text { SD) }\end{array}$ & $27.4 \pm 7.0$ & $10.6 \pm 3.5$ & $<0.0001^{\mathrm{a}}$ \\
\hline
\end{tabular}

$2.5 \mathrm{mmol} / 1$ each), $1 \mu \mathrm{l}$ Recombinant RNasin ${ }^{\circledR}$ Ribonuclease inhibitor (40 U/ $\mu \mathrm{l}), 2 \mu \mathrm{l}$ ImProm-II ${ }^{\mathrm{TM}}$ Reverse Transcriptase (15 U/ $\mu \mathrm{l}$ ) and $12.2 \mu \mathrm{l}$ nuclease-free water to a final volume of $40 \mu \mathrm{l}$. The reaction mixtures were incubated at $70^{\circ} \mathrm{C}$ for $15 \mathrm{~min}$, at $42^{\circ} \mathrm{C}$ for $60 \mathrm{sec}$ and at $25^{\circ} \mathrm{C}$ for $5 \mathrm{~min}$ and the products were stored at $-20^{\circ} \mathrm{C}$.

qRT-PCR was performed in a $20-\mu 1$ reaction containing $10 \mu 1$ 2X QuantiTect SYBR-Green PCR Master mix (Qiagen, Hilden, Germany), $1 \mu 1$ gene-specific primers $(20 \mathrm{mmol} / \mathrm{l})$, $1 \mu \mathrm{l}$ cDNA solution and $8 \mu \mathrm{l}$ nuclease-free water. The reaction mixtures were incubated at $95^{\circ} \mathrm{C}$ for $15 \mathrm{~min}$, followed by 40 cycles at $95^{\circ} \mathrm{C}$ for $10 \mathrm{sec}$, at $60^{\circ} \mathrm{C}$ for $30 \mathrm{sec}$ and at $72^{\circ} \mathrm{C}$ for $30 \mathrm{sec}$, running on a Mx3000 $\mathrm{P}^{\mathrm{TM}}$ thermocycler (Agilent Technologies, Inc., Santa Clara, CA, USA). The primer sequences are presented in Table I.

Statistical analysis. The qRT-PCR data were analyzed by MxPro software (Agilent Technologies, Inc.) and the normalization was performed with U6 small nuclear RNA. The of miRNA contents were calculated using the formula $\Delta \mathrm{Ct}_{\text {miRNA }}=\mathrm{Ct}_{\text {miRNA }}-\mathrm{Ct}_{\mathrm{U} 6}$. A two-sided $\chi^{2}$ test and independent t-tests were used to compare the differences by gender, age, laterality and NSE levels between RB patients and healthy controls. The Mann-Whitney U test was used for the analyses of the expression of different miRNAs. Receiver operating characteristic (ROC) curves were drawn and the areas under the ROC curves (AUCs) were measured to assess the specificity and sensitivity of circulating miRNAs as diagnostic biomarkers for $\mathrm{RB} . \mathrm{P}<0.05$ was considered to indicate a statistically significant difference. Statistical analyses were performed with SPSS 17.0 software (SPSS, Inc., Chicago, IL, USA), the ROC curves were generated by MedCalc 12.7.0.0 (http://www.medcale.org; accessed July 10, 
Table III. Receiver operating characteristic curve are shown for the 3 miRNAs detected in the plasma samples and their combinations with NSE.

\begin{tabular}{|c|c|c|c|c|c|}
\hline $\begin{array}{l}\text { miRNAs, NSE } \\
\text { and combinations }\end{array}$ & AUC & $95 \% \mathrm{CI}$ & Sensitivity (\%) & Specificity (\%) & P-value \\
\hline $\operatorname{miR}-320$ & 0.660 & $0.558-0.752$ & 58 & 74 & 0.0036 \\
\hline miR-let-7e & 0.587 & $0.484-0.684$ & 76 & 42 & 0.1280 \\
\hline miR-21 & 0.548 & $0.446-0.648$ & 46 & 72 & 0.4100 \\
\hline NSE & 0.989 & $0.944-1.000$ & 94 & 100 & $<0.0001$ \\
\hline miR-320 and NSE & 0.996 & $0.957-1.000$ & 98 & 98 & $<0.0001$ \\
\hline miR-let-7e and NSE & 0.991 & $0.948-1.000$ & 94 & 100 & $<0.0001$ \\
\hline miR-21 and NSE & 0.993 & $0.950-1.000$ & 94 & 100 & $<0.0001$ \\
\hline
\end{tabular}

NSE, neuron-specific enolase; AUC, area under the concentration-time curve; CI confidence interval; miRNA, microRNA.

A

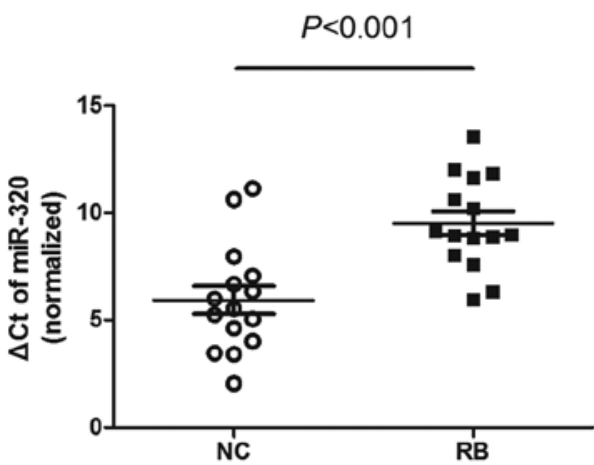

B

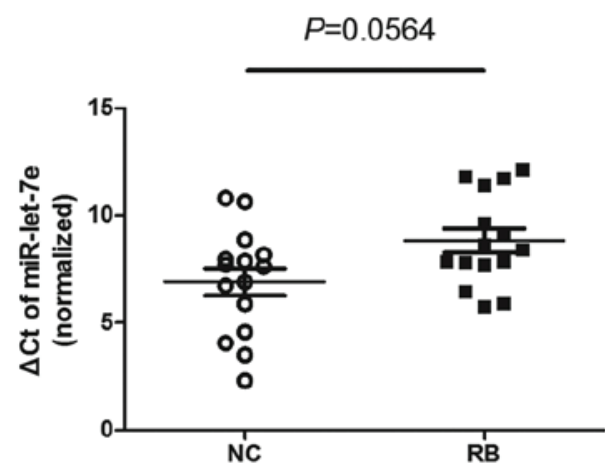

C

$P=0.0475$

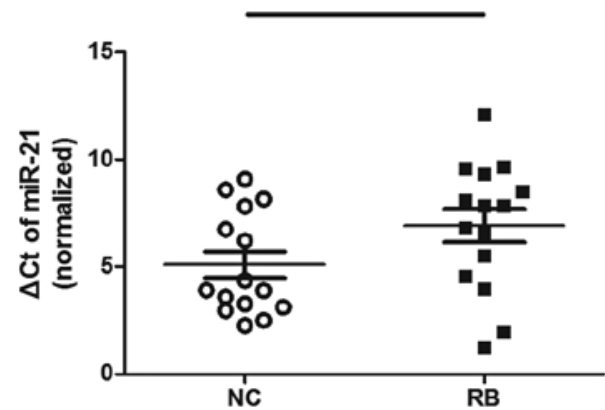

A

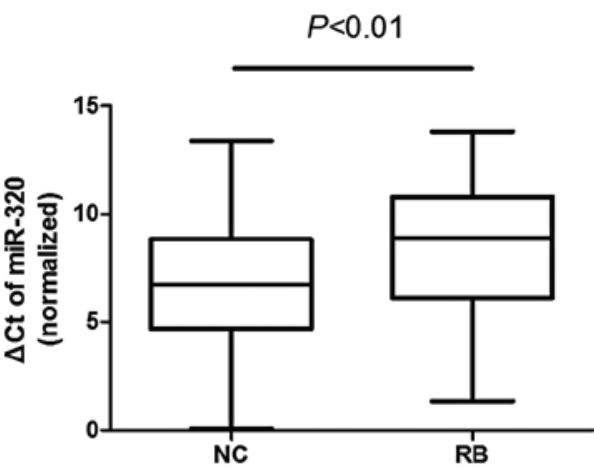

B

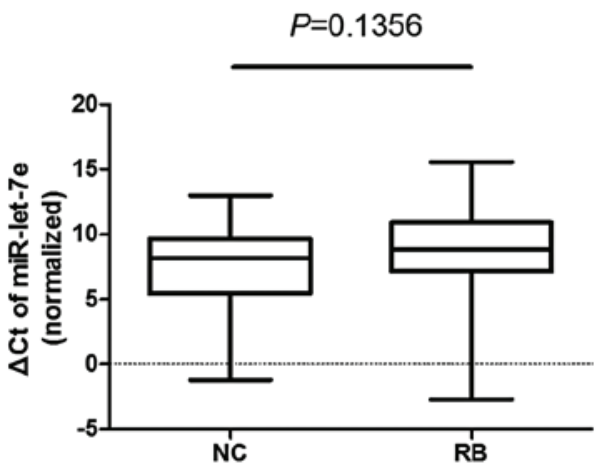

C

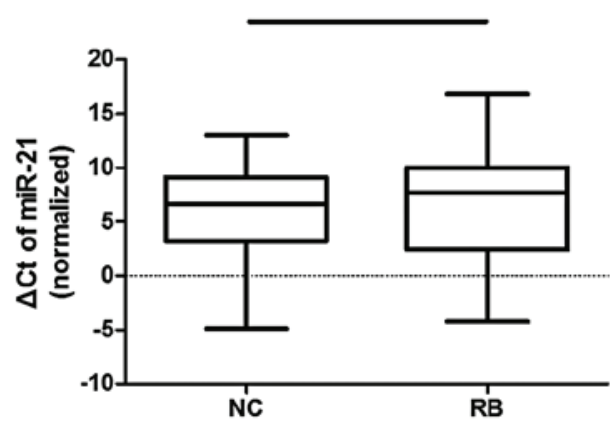

Figure 1. Relative expression levels of plasma miRNAs in initiatory screening. Scatter dot plots of the levels of the 3 plasma miRNAs in retinoblastoma patients $(\mathrm{RB}, \mathrm{n}=15)$ and normal control subjects $(\mathrm{NC}, \mathrm{n}=15)$. The scatter dot plots reflect the plasma levels of (A) miR-320, (B) miR-let-7e and $(\mathrm{C}) \mathrm{miR}-21$. The lines in the scatter dot plots denote the medians. The miRNA plasma levels were lower in RB patients compared to those in controls (higher relative $\Delta \mathrm{Ct}$ to $\mathrm{NC}$ ). miRNA, microRNA.
Figure 2. Relative expression levels of plasma miRNAs in initiatory screening. Box plots of the levels of the 3 plasma miRNAs in retinoblastoma patients $(\mathrm{RB}, \mathrm{n}=50)$ and normal control subjects $(\mathrm{NC}, \mathrm{n}=50)$. The box plots reflect the plasma levels of (A) miR-320, (B) miR-let-7e and (C) miR-21. The lines in the box plots denote the medians. The miRNA plasma levels were lower in $\mathrm{RB}$ patients compared to those in controls (higher relative $\Delta \mathrm{Ct}$ to NC). miRNA, microRNA. 
A

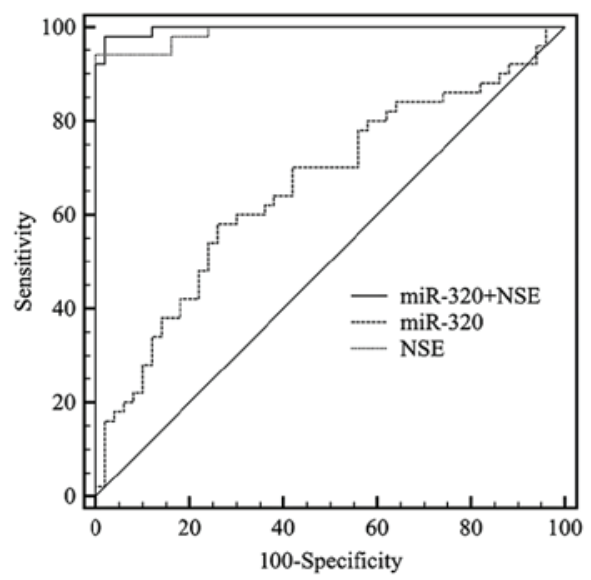

B

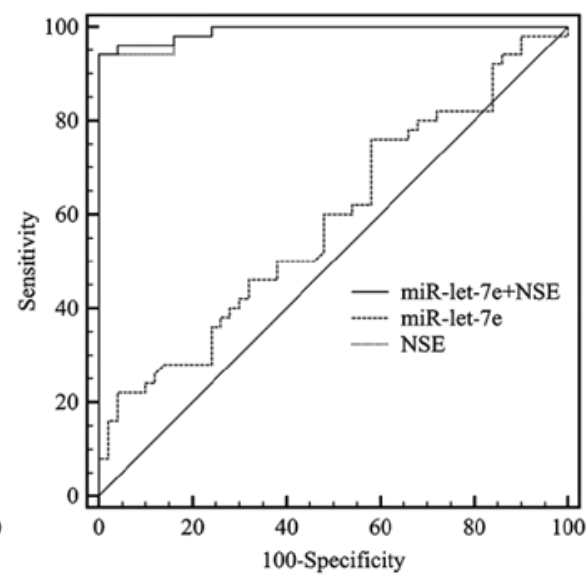

C

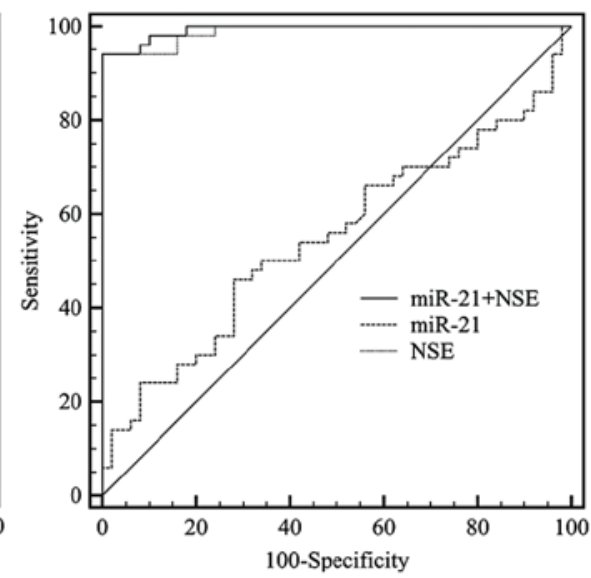

Figure 3. Receiver operating characteristic curves of plasma miRNAs and combined classifiers with NSE. (A) miR-320, (B) miR-let-7e and (C) miR-21 are shown as sparse dotted lines, their combined classifiers with NSE are shown as full lines and the dense dotted lines represent NSE. NSE, neuron-specific enolase. miRNA, microRNA.

2013) and Adobe ${ }^{\circledR}$ Photoshop ${ }^{\circledR}$ CS6 (http://www.adobe.com, accessed February 02, 2013) and the graphs were generated by GraphPad 5.0 software (GraphPad software Inc., La Jolla, CA, USA).

\section{Results}

Patient characteristics. The clinical characteristics of 65 patients diagnosed with RB were recorded and 65 healthy subjects were recruited as controls. The individual characteristics, including gender, age, laterality and clinical stage are summarized in Table II. The NSE levels were significantly higher in the patient group $(27.4 \pm 7.0 \mathrm{ng} / \mathrm{mL})$ compared to those in the control group $(10.6 \pm 3.5 \mathrm{ng} / \mathrm{mL})$. There were no significant differences between the individual characteristics of the patients and those of the control subjects.

Initiatory screening of plasma miRNAs for the detection of $R B$. We measured the different miRNA contents in 30 plasma samples (15 patients and 15 healthy controls). The $\Delta \mathrm{Ct}$ of miR-320 in patient plasma was higher compared to that in the control samples. Similar results were found for miR-let-7e and miR-21 (Fig. 1).

Validation of miR-320, miR-let-7e and miR-21 in a larger sample size. The content of miR-320, miR-let-7e and miR-21 was measured in 100 plasma samples (50 patients and 50 healthy controls). The $\Delta \mathrm{Ct}$ of miR-320 was significantly different between the plasma samples of patients and controls $(\mathrm{P}<0.01)$, whereas the differences in miR-let-7e $(\mathrm{P}=0.1356)$ and miR-21 ( $\mathrm{P}=0.4081)$ were not as significant (Fig. 2).

Diagnostic potential of plasma miRNA and NSE in RB patients. ROC curves were used to assess the diagnostic potential of miR-320, miR-let-7e and miR-21 and AUCs indicated its accuracy and reliability. The AUC of NSE reached 0.989 with a cut-off of 15.9. The AUCs of the 3 miRNAs did not reach $70 \%$ (Table III) and the ROC curves for the combined classifiers (NSE and miRNAs) were significantly improved compared to those for miRNAs alone. However, the performance of the combined classifier was not significantly improved compared to NSE alone (Fig. 3).

\section{Discussion}

NSE is one of the main diagnostic indicators in the earlier stages of RB. In order to test its accuracy and sensitivity, several clinical characteristics were compared between the patients and healthy controls. Only NSE levels presented a significant difference between cases and controls, whereas individual characteristics, such as age and gender, were similar. Of the 65 patients, $>80 \%$ were at IIRC clinical stage D-E via NSE level measurement, which indicated that the NSE level was not superior of RB detection at a very early stage (Table II).

miR-320, miR-let-7e and miR-21 were found to be downregulated in the patient group (Fig. 1) and the plasma levels of these 3 miRNAs were also found to be low when the sample size was expanded to 100 subjects (Fig. 2), with the miR-320 level being significantly lower compared to that in normal subjects. The ROC curves for the miRNAs revealed a weaker diagnostic performance for each miRNA alone, although the P-value was of some value for the diagnosis of $\mathrm{RB}$. The combined classifiers also demonstrated the unreliability. However, an elevation of $0.2-0.7 \%$ significantly lowers the inaccuracy and possibility of misdiagnosis, with a diagnostic value of $98.9 \%$. The plasma miR-320 exhibited the highest diagnostic value among the 3 investigated miRNAs $(\mathrm{P}<0.0001$ : AUC for combined classifier with NSE, 99.6\%) and may be considered as a novel plasma biomarker for the diagnosis of RB.

It was reported that a delayed diagnosis of 6 months of $\mathrm{RB}$ may increase the mortality by $70 \%$ (25); therefore, a biomarker for RB detection at an earlier stage may enable treatment prior to exacerbation, with a lower risk and a higher cure rate. The stability and accuracy of tissue biomarkers are highly associated with the mechanisms underlying tumor development and growth; however, the chances of obtaining a tissue sample when there is no evidence of cancer are limited. Therefore, biomarkers in body fluids are crucial for the early diagnosis of 
cancer and biomarkers in the serum and plasma are increasingly investigated as diagnostic markers. The NSE level was found to be higher in the serum of RB patients compared to those in control subjects and has become one of the most widely used diagnostic tools for the early diagnosis of RB. The accuracy, sensitivity and reliability of NSE have been extensively investigated based on clinical data (26-28).

Serum and plasma miRNAs are considered as potential biomarkers in several types of cancer; however, their performance is not as satisfactory as that of traditional markers, such as NSE, for RB. In the present study, the plasma miR-320, miR-let-7e and miR-21 levels were found to be lower in $\mathrm{RB}$ patients compared to those in healthy control subjects (Fig. 2), whereas their expression in RB tissue was reported to be significantly higher (29). AUC, sensitivity and specificity were not found to be adequate for an accurate prediction on their own. However, combined classifiers with NSE may improve the diagnostic sensitivity and specificity of individual biomarkers to a certain extent, provided that the plasma miRNA levels are of value for the diagnosis of RB. However, further studies are required to assess the reliability and accuracy of miR-320, miR-let-7e and miR-21 as plasma biomarkers of RB.

\section{Acknowledgements}

This study was supported by the General Hospital of the Chinese People's Armed Police Forces (grant no. WZ2010008).

\section{References}

1. Kivelä T: The epidemiological challenge of the most frequent eye cancer: retinoblastoma, an issue of birth and death. Br J Ophthalmol 93: 1129-1131, 2009.

2. Mehta M, Sethi S, Pushker N, Kashyap S, Sen S, Bajaj MS and Ghose S: Retinoblastoma. Singapore Med J 53: 128-135, 2012.

3. Oremek GM, Sauer-Eppel H and Bruzdziak TH: Value of tumour and inflammatory markers in lung cancer. Anticancer Res 27 : 1911-1915, 2007.

4. Hervás Benito I, Rivas Sánchez A, Bello Arques P, et al: Value of 123I-MIBG scanning, neuron-specific enolase and serum ferritin in the diagnosis and follow-up of patients with neuroblastoma. Rev Esp Med Nucl 20: 369-376, 2001 (In Spanish).

5. Wu Z, Mao Y, Yang H, Pan S and Chen Z: The determination of neuron-specific enolase of serum in the diagnosis and supervision of retinoblastoma. Chin J Ophthalmol 34: 117-120, 1998 (In Chinese)

6. Wu Z, Yang H, Pan S and Chen Z: Electrophoretic determination of aqueous and serum neuron-specific enolase in the diagnosis of retinoblastoma. Eye science 13: 12-16, 1997.

7. Kloosterman WP and Plasterk RH: The diverse functions of microRNAs in animal development and disease. Dev Cell 11: 441-450, 2006

8. Stefani $G$ and Slack FJ: Small non-coding RNAs in animal development. Nat Rev Mol Cell Biol 9: 219-230, 2008.

9. Jiang J, Lee EJ, Gusev Y and Schmittgen TD: Real-time expression profiling of microRNA precursors in human cancer cell lines. Nucleic Acids Res 33: 5394-5403, 2005.

10. Chen X, Ba Y, Ma L, et al: Characterization of microRNAs in serum: a novel class of biomarkers for diagnosis of cancer and other diseases. Cell Res 18: 997-1006, 2008.
11. Calin GA and Croce CM: MicroRNA signatures in human cancers. Nat Rev Cancer 6: 857-866, 2006.

12. Gilad S, Meiri E, Yogev Y, et al: Serum microRNAs are promising novel biomarkers. PLoS One 3: e3148, 2008.

13. Mu G, Liu H, Zhou F, Xu X, Jiang H, Wang Y and Qu Y: Correlation of overexpression of HMGA1 and HMGA2 with poor tumor differentiation, invasion, and proliferation associated with let-7 down-regulation in retinoblastomas. Hum Pathol 41 493-502, 2010

14. Motoyama K, Inoue H, Nakamura Y, Uetake H, Sugihara K and Mori M: Clinical significance of high mobility group A2 in human gastric cancer and its relationship to let-7 microRNA family. Clin Cancer Res 14: 2334-2340, 2008.

15. Qian ZR, Asa SL, Siomi H, et al: Overexpression of HMGA2 relates to reduction of the let-7 and its relationship to clinicopathological features in pituitary adenomas. Mod Pathol 22: 431-441, 2009.

16. Rahman MM, Qian ZR, Wang EL, et al: Frequent overexpression of HMGA1 and 2 in gastroenteropancreatic neuroendocrine tumours and its relationship to let-7 downregulation. Br J Cancer 100: 501-510, 2009.

17. Takamizawa J, Konishi H, Yanagisawa K, et al: Reduced expression of the let-7 microRNAs in human lung cancers in association with shortened postoperative survival. Cancer Res 64: 3753-3756, 2004

18. Lawrie CH, Gal S, Dunlop HM, et al: Detection of elevated levels of tumour-associated microRNAs in serum of patients with diffuse large B-cell lymphoma. Br J Haematol 141: 672-675, 2008.

19. Hsieh IS, Chang KC, Tsai YT, et al: MicroRNA-320 suppresses the stem cell-like characteristics of prostate cancer cells by downregulating the Wnt/beat-catenin signaling pathway. Carcinogenesis 34: 530-538, 2013.

20. Zhao JJ, Yang J, Lin J, et al: Identification of miRNAs associated with tumorigenesis of retinoblastoma by miRNA microarray analysis. Childs Nerv Syst 25: 13-20, 2009

21. Chan SH, Wu CW, Li AF, Chi CW and Lin WC: miR-21 microRNA expression in human gastric carcinomas and its clinical association. Anticancer Res 28: 907-911, 2008.

22. Kumar S, Keerthana R, Pazhanimuthu A and Perumal P: Overexpression of circulating miRNA-21 and miRNA-146a in plasma samples of breast cancer patients. Indian J Biochem Biophys 50: 210-214, 2013.

23. Toiyama Y, Takahashi M, Hur K, et al: Serum miR-21 as a diagnostic and prognostic biomarker in colorectal cancer. J Natl Cancer Inst 105: 849-859, 2013.

24. Li Y, Li W, Ouyang Q, Hu S and Tang J: Detection of lung cancer with blood microRNA-21 expression levels in Chinese population. Oncol Lett 2: 991-994, 2011

25. Dimaras H, Kimani K, Dimba EA, Gronsdahl P, White A, Chan HS and Gallie BL: Retinoblastoma. Lancet 379: 1436-1446, 2012.

26. Kivelä T: Neuron-specific enolase in retinoblastoma: An immunohistochemical study. Acta Ophthalmol (Copenh) 64: 19-25, 1986.

27. Nakajima T, Kato K, Kaneko A, et al: High concentrations of enolase, alpha- and gamma-subunits, in the aqueous humor in cases of retinoblastoma. Am J Ophthalmol 101: 102-106, 1986.

28. Nucci P, Tredici G, Manitto MP, et al: Neuron-specific enolase in ophthalmology. Arch Ital Anat Embriol 96: 73-76, 1991 (In Italian).

29. Beta M, Venkatesan N, Vasudevan M, Vetrivel U, Khetan V and Krishnakumar S: Identification and in silico analysis of retinoblastoma serum microRNA profile and gene targets towards prediction of novel serum biomarkers. Bioinform Biol Insights 7: 21-34, 2013. 